\title{
Modeling the effects of cyclodextrin on intracellular membrane vesicles from Cos-7 cells prepared by sonication and carbonate treatment
}

Peter Kilbride, Holly J. Woodward, Kuan Boone Tan, Nguyễn T.K. Thanh, K.M. Emily Chu, Shane Minogue, Mark G Waugh

Cholesterol has important functions in the organization of membrane structure and this may be mediated via the formation of cholesterol-rich, liquid-ordered membrane microdomains often referred to as lipid rafts. Methyl-beta-cyclodextrin (cyclodextrin) is commonly used in cell biology studies to extract cholesterol and therefore disrupt lipid rafts. However, in this study we reassessed this experimental strategy and investigated the effects of cyclodextrin on the physical properties of sonicated and carbonate-treated intracellular membrane vesicles isolated from Cos-7 fibroblasts. We treated these membranes, which mainly originate from the trans-Golgi network and endosomes, with cyclodextrin and measured the effects on their equilibrium buoyant density, protein content, represented by the palmitoylated protein phosphatidylinositol 4-kinase type Ilalpha, and cholesterol. Despite the reduction in mass stemming from cholesterol removal, the vesicles became denser, indicating a possible large volumetric decrease, and this was confirmed by measurements of hydrodynamic vesicle size. Subsequent mathematical analyses demonstrated that only half of this change in membrane size was attributable to cholesterol loss. Hence, the non-selective desorption properties of cyclodextrin are also involved in membrane size and density changes. These findings may have implications for preceding studies that interpreted cyclodextrin-induced changes to membrane biochemistry in the context of lipid raft disruption without taking into account our finding that cyclodextrin treatment also reduces membrane size. 
1 Modeling the effects of cyclodextrin on intracellular membrane vesicles from Cos-7 cells

2 prepared by sonication and carbonate treatment

3 Authors: $\quad$ Peter Kilbride*, Holly J. Woodward*, Kuan B. Tan$ Nguyễn T. K. Thanh\#, K.M.

4 Emily Chu*, Shane Minogue* and Mark G. Waugh*\$

5

Address $\quad *$ UCL Institute for Liver \& Digestive Health

Royal Free Campus,

University College London

9

Rowland Hill Street

London NW3 2PF

11

United Kingdom

12

\#Biophysics Group

Department of Physics \& Astronomy

University College London

Gower Street,

London WC1E 6BT

United Kingdom \&

UCL Healthcare Biomagnetic and Nanomaterials Laboratories

21 Albemarle Street,

London W1S 4BS

United Kingdom 
24 \$Corresponding author; e-mail: m.waugh@ucl.ac.uk

25 Running Title: Membrane size and cholesterol

27 Abbreviations: Cyclodextrin - methyl- $\beta$-cyclodextrin, DMEM - Dulbecco's Modified Eagle's

28 Medium, ECL - enhanced chemiluminescence, HRP - horseradish peroxidase, PBS - phosphate-

29 buffered saline, OCRL - oculocerebrorenal syndrome of Lowe, PI4KII $\alpha$ - phosphatidylinositol 4-

30 kinase type II $\alpha$, PVDF - polyvinylidene difluoride, SDS-PAGE - sodium dodecyl sulfate-

31 polyacrylamide gel electrophoresis, TGN - trans-Golgi network.

32 Keywords: Cholesterol; cyclodextrin; lipid raft; membrane; TGN, PI 4-kinase. 


\section{Abstract}

Cholesterol has important functions in the organization of membrane structure and this may be mediated via the formation of cholesterol-rich, liquid-ordered membrane microdomains often referred to as lipid rafts. Methyl- $\beta$-cyclodextrin (cyclodextrin) is commonly used in cell biology studies to extract cholesterol and therefore disrupt lipid rafts. However, in this study we reassessed this experimental strategy and investigated the effects of cyclodextrin on the physical properties of sonicated and carbonate-treated intracellular membrane vesicles isolated from Cos-7 fibroblasts. We treated these membranes, which mainly originate from the transGolgi network and endosomes, with cyclodextrin and measured the effects on their equilibrium buoyant density, protein content, represented by the palmitoylated protein phosphatidylinositol 4-kinase type Il $\alpha$, and cholesterol. Despite the reduction in mass stemming from cholesterol removal, the vesicles became denser, indicating a possible large volumetric decrease, and this was confirmed by measurements of hydrodynamic vesicle size. Subsequent mathematical analyses demonstrated that only half of this change in membrane size was attributable to cholesterol loss. Hence, the non-selective desorption properties of cyclodextrin are also involved in membrane size and density changes. These findings may have implications for preceding studies that interpreted cyclodextrin-induced changes to membrane biochemistry in the context of lipid raft disruption without taking into account our finding that cyclodextrin treatment also reduces membrane size. 
53

54

\section{Introduction}

In this study we investigated the relationship between membrane composition, density, and size by using methyl- $\beta$-cyclodextrin (cyclodextrin) to rapidly deplete membrane cholesterol from an isolated intracellular membrane preparation. Cyclodextrins are a family of cyclic oligosaccharides that adopt a cone-like structure in aqueous solution, with an internal hydrophobic core that can sequester lipids from membranes (Heine et al., 2007; Pinjari et al., 2006). Cyclodextrins have useful pharmaceutical applications as soluble carriers for hydrophobic molecules and are also commonly used in biochemical and cell biology studies to manipulate membrane lipid levels (Loftsson and Brewster, 1996; Rodal et al., 1999; Welliver, 2006; Zidovetzki and Levitan, 2007). Cyclodextrin efficaciously removes sterols such as cholesterol from biological membranes but can also remove other lipids such as sphingomyelin and phosphatidylcholine (Ottico et al., 2003). Recently cyclodextrin and the related molecule hydroxypropyl- $\beta$-cyclodextrin have been shown to alleviate the pathological intracellular accumulation of free cholesterol in Niemann-Pick Type C disease models (Camargo et al., 2001; Davidson et al., 2009; Holtta-Vuori et al., 2002; Lim et al., 2006; Liu et al., 2008; Liu et al., 2010; Liu et al., 2009; Mbua et al., 2013; Pontikis et al., 2013; Ramirez et al.; Ramirez et al., 2010; Rosenbaum et al., 2010; Swaroop et al., 2012; te Vruchte et al., 2014; Vance and Karten, 2014; Vite et al., 2015; Waugh, 2015). These recent developments demonstrate a potential therapeutic use for cyclodextrins and also clearly establish their efficacy for reducing the cholesterol content of endosomal membranes (Rosenbaum et al., 2010; Shogomori and Futerman, 2001). In addition, we have previously reported that the addition of cyclodextrin to cultured cells leads to the vesicularization and contraction of the trans-Golgi network (TGN) and endosomal membranes (Minogue et al., 2010). These cyclodextrin-induced changes to 
intracellular biomembrane architecture are associated with alterations to intramembrane

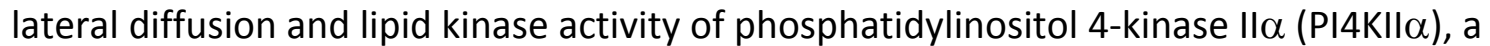
constitutively palmitoylated and membrane-associated enzyme (Barylko et al., 2009; Lu et al., 2012) that may be important in the etiology of some cancers and neurodegenerative disorders (Chu et al., 2010; Clayton et al., 2013a; Li et al., 2010; Li et al., 2014; Simons et al., 2009; Waugh, 2012, 2014, 2015).

Whilst cyclodextrin has been mainly used to remove cholesterol from the plasma membrane our focus here is on characterizing the effects of such treatment on intracellular membranes where cholesterol levels are known to be important for processes such as protein sorting and trafficking from the TGN (Paladino et al., 2014). Since the effects of cyclodextrin on intracellular membranes are important to understand both in a disease context (Vite et al., 2015) and for furthering our knowledge about the functions of cholesterol on intracellular membranes, we decided to investigate more comprehensively how cyclodextrin alters the biophysical properties of a lipid-raft-enriched membrane fraction isolated from intracellular TGN and endosomal membranes (Minogue et al., 2010; Waugh et al., 2011a). In particular, we sought to understand more fully the cyclodextrin-induced changes to the equilibrium buoyant densities of isolated cholesterol-rich membrane fractions that we and others have reported in a number of preceding publications (for examples, see (Hill et al., 2002; Kabouridis et al., 2000; Matarazzo et al., 2012; Minogue et al., 2010; Navratil et al., 2003; Pike and Miller, 1998; Spisni et al., 2001; Xu et al., 2006; Zidovetzki and Levitan, 2007)). In these previous experiments, cholesterol depletion with cyclodextrin rendered the membrane fraction less buoyant, leading to the cyclodextrin-treated membranes banding to a denser region in an equilibrium density gradient. This cyclodextrin-induced change, sometimes referred to as a density shift, has 
99 allowed us to design, using sucrose density gradients, a membrane floatation assay in which we

100 have been able to separate cholesterol-replete and -depleted membranes before and after

101 cyclodextrin treatment.

102 In many of these prior studies, a cyclodextrin-dependent redistribution of biomolecules to a

103 denser membrane fraction was interpreted as a delocalization from cholesterol-rich lipid rafts

104 or liquid-ordered domains to a less buoyant, liquid-disordered, non-raft fraction. This reasoning

105 stems from the idea that raft-enriched membrane domains are intrinsically buoyant due to

106 their high lipid-to-protein ratio. However, since density is defined as mass divided by volume

107 we reassessed these inferences on the grounds that in the absence of a membrane volume

108 change, a reduction in mass alone would result in a membrane becoming more buoyant, i.e.

109 less dense.

110 To explore the relationship between cholesterol content and membrane density, we employed

111 our membrane floatation assay to measure the change in the physical properties and

112 biochemical composition of cholesterol-enriched membrane vesicles following cyclodextrin

113 treatment. We then analyzed these changes to mathematically model, from first principles, the

114 degree to which the mass and volume of the membrane domains would have to alter in order

115 to account for the measured change in membrane density. Finally, we provide a mathematical

116 solution to explain the relationship between membrane cholesterol mass and vesicle density.

\section{Materials and Methods}

118 Materials. All cell culture materials, enhanced chemiluminescence (ECL) reagents and X-ray film

119 were purchased from GE Healthcare Life Sciences, UK. Polyvinylidene difluoride (PVDF)

120 membrane was bought from Merck Millipore UK. Horseradish peroxidase (HRP)-linked 
121 secondary antibodies were purchased from Cell Signalling Technology UK. The antibody to

122 PI4KIl $\alpha$ was previously described by us (Minogue et al., 2010). HRP-linked cholera toxin B

123 subunit was purchased from Sigma-Aldrich UK. Sucrose was obtained from VWR International

124 Ltd UK. Complete protease inhibitor tablets were purchased from Roche Ltd UK. All other

125 reagents were from Sigma-Aldrich UK

126 Cell culture. Cos-7 cells obtained from the European Collection of Cell Cultures operated by

127 Public Health England were maintained at $37^{\circ} \mathrm{C}$ in a humidified incubator at $10 \% \mathrm{CO}_{2}$. Cells were 128 cultured in Dulbecco's Modified Eagle's Medium (DMEM) supplemented with Glutamax, 10\%

129 fetal calf serum, 50 i.u./mL penicillin, and $50 \mu \mathrm{g} / \mathrm{mL}$ streptomycin. Cell monolayers were grown

130 to confluency in $10 \mathrm{~cm}$ tissue culture dishes. Typically, four confluent plates of cells were used

131 in each subcellular fractionation experiment.

132 Subcellular fractionation by sucrose density gradient centrifugation. A buoyant subcellular

133 fraction enriched for TGN and endosomal membranes was prepared according to our

134 previously published method (Minogue et al., 2010; Waugh et al., 2006). Confluent cell

135 monolayers were washed twice in ice-cold phosphate-buffered saline (PBS) pH 7.4 and then

136 scraped into $2 \mathrm{~mL}$ of homogenization buffer (Tris-HCl 10mM, EGTA $1 \mathrm{mM}$, EDTA $1 \mathrm{mM}$, sucrose

$137250 \mathrm{mM}$, plus Complete ${ }^{\mathrm{TM}}$ protease inhibitors, pH 7.4). Post-nuclear supernatants were

138 prepared by Dounce homogenization of the cells suspended in homogenization buffer followed

139 by centrifugation at $1,000 \mathrm{~g}$ at $4^{\circ} \mathrm{C}$ for $2 \mathrm{~min}$ to pellet nuclei and unbroken cells. Cellular

140 organelles were separated by equilibrium density gradient centrifugation by overnight

141 ultracentrifugation on a $12 \mathrm{~mL}, 10-40 \% \mathrm{w} / \mathrm{v}$ sucrose density gradient as previously described

142 (Waugh et al., 2003a; Waugh et al., 2003b; Waugh et al., 2006). Using this procedure, a buoyant 
143 TGN-endosomal enriched membrane fraction consistently banded in gradient fractions 9 and 10

144 and was harvested as described before (Waugh et al., 2003b; Waugh et al., 2006).

145 Refractometry to measure membrane density. The refractive index of each membrane fraction

146 was determined using a Leica AR200 digital refractometer. Refractive index values were then

147 converted to sucrose densities using Blix tables (Dawson et al., 1986) and linear regression

148 carried out using GraphPad Prism software.

150

151

152

\section{Membrane floatation assay to measure the equilibrium buoyant density of membrane} vesicles. This assay was previously described by us (Minogue et al., 2010). Briefly, $400 \mu \mathrm{L}$ of cyclodextrin (20 mM) dissolved in water was added to an equal volume of TGN/endosomal membranes on ice for $10 \mathrm{~min}$ to give a cyclodextrin concentration of $10 \mathrm{mM}$. Then, $200 \mu \mathrm{L}$ of sodium carbonate $(0.5 \mathrm{M}, \mathrm{pH} 11.0)$ was added to a final concentration of $50 \mathrm{mM}$ in a $1 \mathrm{~mL}$ sample. The carbonate-treated membranes were probe-sonicated on ice using a VibraCell probe sonicator from Sonics \& Materials Inc, USA at amplitude setting 40 in pulsed mode for $3 \mathrm{x}$ $2 \mathrm{sec}$ bursts. To the $1 \mathrm{~mL}$ sonicated membrane samples, $3 \mathrm{~mL}$ of $53 \% \mathrm{w} / \mathrm{v}$ sucrose in $\mathrm{Tris}-\mathrm{HCl} 10$ $\mathrm{mM}$, EDTA $1 \mathrm{mM}$ and EGTA $1 \mathrm{mM}, \mathrm{pH} 7.4$ was added to form $4 \mathrm{~mL}$ of sample in $40 \% \mathrm{w} / \mathrm{v}$ sucrose and a sodium carbonate concentration of $12.5 \mathrm{mM}$ and, where applicable, a cyclodextrin concentration of $2 \mathrm{mM}$. A discontinuous sucrose gradient was formed in a $12 \mathrm{~mL}$ polycarbonate tube by overlaying the $40 \%$ sucrose layer with $4 \mathrm{~mL} 35 \% \mathrm{w} / \mathrm{v}$ and $4 \mathrm{~mL} 5 \% \mathrm{w} / \mathrm{v}$ sucrose in Tris- $\mathrm{HCl} 10 \mathrm{mM}$, EDTA $1 \mathrm{mM}$ and EGTA $1 \mathrm{mM}, \mathrm{pH}$ 7.4. The gradient was centrifuged overnight at $185,000 \mathrm{~g}$ at $4^{\circ} \mathrm{C}$ in a Beckman LE-80K ultracentrifuge and $12 \times 1 \mathrm{~mL}$ fractions were harvested beginning at the top of the tube.

Immunoblotting of sucrose density gradient fractions. The protein content of equal volume aliquots of each density gradient fraction was separated by sodium dodecyl sulfate- 
166 polyacrylamide gel electrophoresis (SDS-PAGE), transferred to PVDF membranes and probed

167 with antibodies directed against proteins of interest. Western blots were visualized by

168 chemiluminescence and bands were quantified from scanned X-ray films using image analysis

169 software in Adobe Photoshop CS4.

170 Measurements of membrane lipid levels. The cholesterol content of equal volume membrane

171 fractions was assayed using the Amplex red cholesterol assay kit (Molecular Probes). The use of

172 this assay to measure membrane cholesterol mass has been previously validated (Bate et al.,

173 2008; Minogue et al., 2010; Nicholson and Ferreira, 2009). Ganglioside glycosphingolipids were

174 detected by dot blotting of membrane fractions (Ilangumaran et al., 1996) and probing with

175 HRP-conjugated cholera toxin B subunit as described previously (Ilangumaran et al., 1996;

176 Mazzone et al., 2006; Waugh, 2013; Waugh et al., 2011a; Waugh et al., 2011b). Membrane-

177 bound cholera toxin was visualized by incubation with chemiluminescence detection reagents

178 and spots were quantified as described for the analysis of immunoblotting data (Waugh, 2013).

179

180

181

182

183

\section{Dynamic light scattering measurement to measure hydrodynamic diameter of membrane} vesicles. The hydrodynamic size of the membrane vesicles in the gradient fraction was studied with a Zetasizer Nano ZS90 (Malvern Instruments). All diluted samples were prepared in filtered $(0.2 \mu \mathrm{m})$ Millipore $\mathrm{ddH}_{2} \mathrm{O}$ to avoid sample artifacts, and measurements were made at $25^{\circ} \mathrm{C}$ in triplicate.

\section{Mathematical modelling of membrane compositional changes.}

Nomenclature

$\rho=$ mass density $(\mathrm{kg} / \mathrm{L})$ 
$187 \quad V$ = volume $(\mathrm{L})$

188 Subscripts are used to specify a unit being examined, with $s$ and $r$ defining treatment sensitive

189 (assuming that most of this fraction is composed of cholesterol with a density of around $\rho=$

1901.067 ) and remaining components, respectively. The subscript -post is used to denote values

191 for vesicles post cyclodextrin treatment.

192 The fractions are not considered discrete sections of the vesicles; rather they can be mixed and

193 inter-connected.

194 The mass density of a particle is defined as the mass per unit volume. To determine the mass

195 density of an object consisting of multiple discrete components in a steady state, a linear

196 combination of its components can be used as in equation [1].

$198 \rho=\frac{m_{1}+m_{2}+m_{3}+\ldots}{V_{1}+V_{2}+V_{3}+\ldots}$

201 where the subscripts denote the mass and volume of the separate components. Through

202 normalizing the total volume $\mathrm{V}=\mathrm{V}_{1}+\mathrm{V}_{2}+\mathrm{V}_{3}+\ldots=1$, the density can simplify to

$\rho=m_{1}+m_{2}+m_{3}+\ldots m_{n}$ 
204 where $m_{n}$ now refers to the mass of the volume fraction in question. Considering an object

205 composed of $\mathrm{n}$ different materials the overall mass density is therefore

$\rho_{\text {whole }}=\rho_{\text {fraction } 1} V_{\text {fraction } 1}+\rho_{\text {fraction } 2} V_{\text {fraction } 2}+\rho_{\text {fraction } 3} V_{\text {fraction } 3}+\ldots$

$206=\sum_{j=1}^{j=n} \rho_{j} V_{j}$

208 where $\rho_{\mathrm{j}}$ is the density of fraction $\mathrm{j}$, and $\mathrm{v}_{\mathrm{j}}$ is the volume fraction of material $\mathrm{j}$.

209 To determine the \% composition of the vesicles, boundary conditions were formulated and

210 solved using simultaneous equations. Pre-treatment, the system was described through

211 Equation [3]:

212

$213 V_{r} \times \rho_{r}+V_{s} \times \rho_{s}=\rho_{\text {pre }}$

215 where the fractional volume of the residual component is given by $V_{r}$, the fractional volume of

216 the treatment sensitive component $=\mathrm{V}_{\mathrm{s}}$, and $\rho_{\text {pre }}$ was the measured density of the vesicle pre-

217 treatment. 
218 A second simultaneous equation arises through the physical definition of the system, which is

219 the total volume has been normalised to one:

220

$$
V_{r}+V_{s}=1
$$

221

\section{[4]}

222

i.e. combining all the fractions in a vesicle together equaled fraction one of a vesicle.

223 The $3^{\text {rd }}$ simultaneous equation was determined with respect to the post-treatment density. It

224 can be derived that for the cyclodextrin sensitive fraction:

228 While the mass and volume of the cholesterol fraction change, its intrinsic density does not.

$231 \quad \frac{m_{\text {pre }}}{V_{\text {pre }}}=\frac{m_{\text {post }}}{V_{\text {post }}} \Rightarrow V_{\text {post }}=\frac{m_{\text {post }}}{m_{\text {pre }}} \times V_{\text {pre }}$ 
$234 \frac{m_{\text {post }}}{m_{\text {pre }}}=$ the mass post-treatment relative to the pre-treatment mass, which was defined as the

235 dimensionless parameter $\mathrm{M}$. The RHS of Equation [6] then simplified to: $\mathrm{V}_{\text {s-pre }} \times \mathrm{M}$. A similar

236 procedure was followed for $\mathrm{V}_{\mathrm{r}}$.

237 In order to define the mass density of the vesicles post-treatment, equation [3] was modified 238 and normalized to take account of the change of mass. This yielded:

$239 \rho_{\text {post }}=\frac{V_{r} \times \rho_{r}+V_{s} \times \rho_{s} \times M_{s-\text { post }}}{V_{r}+V_{s} \times M_{s-p o s t}}$

242 Statistical analysis. Data are presented as mean \pm SEM of at least three determinations.

243 Statistical significance was assessed using the two-tailed student $t$ test and $P$ values $<0.05$ were 244 deemed to be statistically significant.

\section{Results}

Changes in membrane composition and density following cholesterol depletion. The starting material for this set of experiments was our previously characterized cholesterol-rich intracellular membrane fraction prepared from post-nuclear cell supernatants. These 
250

251

252

253

254

255

256

257

258

259

260

261

262

263

264

265

266

267

268

269

270

271

272

membranes were isolated on equilibrium sucrose density gradients and their identity as a TGNendosomal fraction was confirmed by Western blotting for PI4KIl $\alpha$ and syntaxin-6 (Minogue et al., 2010; Waugh et al., 2006). To investigate in more detail the relationship between cholesterol levels, membrane composition and membrane biophysical properties, we employed our recently described floatation assay method to determine the equilibrium buoyant densities of TGN-endosomal membrane domains using sucrose gradients (see work flow chart in Figure 1). This technique involved treating the membranes with cyclodextrin ( $10 \mathrm{mM}$ ) for $10 \mathrm{~min}$ to extract cholesterol followed by probe sonication to induce their vesicularization and fragmentation (Waugh et al., 1999; Waugh et al., 1998). The sonication step was carried out in alkaline carbonate buffer which is a well-established means for removing peripheral proteins including actin from membranes (Fujiki et al., 1982; Nebl et al., 2002). This procedure was necessary in light of the extensive literature demonstrating that peripherally associated membrane proteins can influence membrane architecture, geometry and density, and such additional heterogeneity in these membrane characteristics could potentially complicate subsequent biophysical analyses. This combination of probe sonication and carbonate addition was aimed at generating a population of membrane vesicles stripped of peripheral proteins including cyclodextrin-sensitive cytoskeletal proteins which have the potential to modify membrane microdomain stability [46-49]. Furthermore, the inclusion of these treatments meant that the integral protein and lipid compositions of the vesicles would be the principal determinants of membrane density.

In this set of experiments we compared the effects of cyclodextrin treatment on the biochemical composition of the buoyant (fractions 5 to 8 ) and dense (fractions 9 to 12) regions of the sucrose gradient. Cyclodextrin addition resulted in a large $(83.4 \pm 2.75 \%, n=3)$ decrease 
273 in the cholesterol mass of the buoyant fractions protein without any significant accumulation in 274 the denser region of the sucrose gradient (Figure 2). This large reduction in cholesterol also 275 coincided with a relocalization of the membrane-associated PI4KII $\alpha$ protein to denser

276 membrane fractions 9 to 12 (Figure 2). We quantified this change in PI4KIl $\alpha$ distribution, which 277 was also noted in our previous publication (Minogue et al., 2010), and found that unlike the 278 situation with cholesterol, cyclodextrin did not result in an overall loss of PI4KII $\alpha$ from the 279 membrane fractions.

We used refractometry to measure the sucrose density of the gradient fractions. Trial experiments revealed that the final, diluted cyclodextrin concentrations of $200 \mathrm{mM}$ present in the dense gradient fractions did not impact on the refractive index readings for these samples. These measurements allowed us to determine that the inclusion of cyclodextrin caused the main protein fraction to shift in density from 1.096 to $1.126 \mathrm{~g} / \mathrm{mL}$ (Figure 3).

Finally, we measured cyclodextrin-effected changes to the hydrodynamic diameter of the vesicles by dynamic light scattering. Even though the isolated membrane vesicles were found to be heterogeneous we focused on a peak signal corresponding to a vesicle population in the biologically relevant size range of $10-1000 \mathrm{~nm}$. We ascertained that while there was no change in the total number of vesicles, the average vesicle diameter shrunk from 780 to $42 \mathrm{~nm}$ in the buoyant fraction and from 453 to $271 \mathrm{~nm}$ in the dense fraction (Table 1). These results showed that the reduction in cholesterol levels brought about by cyclodextrin treatment caused the membrane vesicle sizes to decrease considerably.

Together, these experiments revealed that cholesterol depletion with cyclodextrin resulted in a reduction in membrane buoyancy, as evidenced by the delocalization of PI4KIl $\alpha$-containing membranes to a denser region of the sucrose gradient and also a reduction in membrane size. 
296 Therefore, we decided to mathematically model the relationship between these different

297 parameters.

298

299 Mathematical Modeling

300 In this model, we determined an expected value for vesicle size post cyclodextrin treatment in

301 our system and compared it with experimental data. From our experimental measurements,

302 there was an $83.4 \pm 2.75 \%$, reduction in the cyclodextrin sensitive cholesterol component with

303 other components not directly affected by the treatment. The total increase in mass density of

304 the vesicles through cyclodextrin treatment was known (from $1.096 \pm 0.003 \mathrm{mg} / \mathrm{mL}$ for fraction

3055 to $1.122 \pm 0.0005 \mathrm{mg} / \mathrm{mL}$ for fraction 10$)$. The $\%$ composition of these two components and

306 the density of the non-cholesterol residual component were unknown and approximated in this

307 work, based on the above assumptions.

308 To determine the volumetric fractional composition of the vesicles pre cyclodextrin treatment

309 and the density of the residual component, the experimentally measured values were inserted

310 into equations 4,6, and 7, giving equations 8-10:

311

312

$V_{r} \times \rho_{r}+V_{s} \times 1.067=1.096 \pm 0.003$

313 [8]

$314 V_{r}+V_{s}=1$

315 [9] 
$V_{r} \times \rho_{r}+V_{s} \times 1.067 \times(0.166 \pm 0.00275)$$$
V_{r}+V_{s} \times(0.166 \pm 0.00275)
$$

317 [10]

318

319 Calculating these equations [8] to [10] allowed us to predict the volume fractions of the vesicle pretreatment as follows - cholesterol $0.567 \pm 0.072(56.7 \pm 7.2 \%)$, residual component $0.433 \pm$ $0.072(43.3 \pm 7.2 \%)$, and a density of the residual component of $1.134 \pm 0.005 \mathrm{mg} / \mathrm{mL}$. As liquidordered domains typically comprises $20-30 \%$ cholesterol, the higher than expected value determined here is most likely the result of some membrane components being removed during the membrane isolation procedure and particularly by the alkaline carbonate addition step, leading to an apparent enrichment of cholesterol in the isolated fraction. Hence, the \% value for cholesterol determined here is not the physiological proportion of cholesterol in TGN/endosomal membranes but rather, the amount present in the membrane vesicles after the extensive membrane disruption and isolation procedures used in this study. In concordance with this explanation, we have previously shown that membrane fractions prepared in the presence of carbonate are subject to substantial depletion of non-integral proteins (Waugh, 2013; Waugh et al., 2011a; Waugh et al., 2011b). As proteins have a density of $1.35 \mathrm{mg} / \mathrm{mL}$

332 (Chick and Martin, 1913; Fischer et al., 2004) and other membrane components such as lipids tend to have much lower densities, the value of $1.134 \pm 0.005 \mathrm{mg} / \mathrm{mL}$ calculated for the density of the residual component seems reasonable.

Cyclodextrin treatment resulted in the total amount of cholesterol in the system to be reduced 336 by $83.4 \%$; however, the absolute volumes of the other components remained constant. To 
337 calculate the volume concentrations post cyclodextrin, three more simultaneous equations

338 were formulated and solved by the same method:

339

340

$V_{r-p o s t} \times \rho_{r}+V_{s-p o s t} \times 1.067=1.122 \pm 0.0005$

341

[11]

342

$V_{r-p o s t}+V_{s-p o s t}=1$

343

[12]

344

$\frac{V_{r-\text { post }} \times \rho_{r}+V_{s-\text { post }} \times 1.067 \times 6.02}{V_{r-\text { post }}+V_{s-\text { post }} \times 6.02}=1.096 \pm 0.03$

The predicted vesicle composition post treatment obtained by solving any two of equations [11]

347 to [13] was: cholesterol $0.179 \pm 0.047(17.9 \pm 4.7 \%)$ and residual component $0.821 \pm 0.047$ (82.1 $348 \pm 4.7 \%)$.

349

350 The relative volume of the treated vesicles was calculated through equation [14]:

$V_{r}+V_{s} \times 0.166 \pm 0.0275=$ New Volume 
355 giving a relative volume of $0.527 \pm 0.073$, i.e. post treatment, the vesicle was $49.1 \pm 7.3 \%$ of its

356 original size. This corresponds to the diameter of the treated vesicles of $0.81 \pm 0.04$, i.e. the

357 radius must have shrunk by $19 \pm 4 \%$.

358

359

Experimental measurements showed the diameter of the vesicles falling from $453 \pm 177.1 \mathrm{~nm}$

to $270.2 \pm 68.8 \mathrm{~nm}$ post treatment, a $40.4 \%$ decline in diameter and thus an $88.8 \%$ fall in vesicle volume. This differs markedly from what has been calculated based on cyclodextrin affecting cholesterol alone and is consistent with previous work demonstrating that cyclodextrin can also sequester a range of hydrophobic molecules (reviewed in (Zidovetzki and Levitan, 2007)). These

results imply that only about $50 \%$ of the change in membrane size is due to cholesterol desorption.

Since the mathematical analysis demonstrated that the decrease in membrane size could not

be fully accounted for by cholesterol loss, we investigated the effect of cyclodextrin addition on the levels of membrane gangliosides which are glycosphingolipids that are structurally unrelated to sterols. Changes in ganglioside lipid distribution were determined using HRPconjugated cholera toxin B subunit as a probe. Kuziemko and colleagues (Kuziemko et al., 1996) previously determined that Cholera-toxin binds to gangliosides in the order GM1 > GM2 $>$ GD1A > GM3 > GT1B > GD1B > asialo-GM1, albeit with a > 200 fold difference in binding affinity between GM1 and asialo-GM1. Therefore, unlike thin layer chromatography, dotblotting immobilized sucrose density gradient fractions with cholera toxin B subunit does not permit the separation and quantitation of individual glycosphingolipid species. Furthermore there is a possibility that in addition to gangliosides, the toxin may also bind to the carbohydrate moieties of glycosylated proteins associated with the isolated vesicles (Blank et 
378 al., 2007; Uesaka et al., 1994). Bearing in mind these limitations, we used this well established

379 technique (Clarke et al., 2007; Correa et al., 2007; Domon et al., 2011; Ersek et al., 2015;

380 Ilangumaran et al., 1996; Liu et al., 2013; Liu et al., 2015; Mazzone et al., 2006; Nguyen et al.,

381 2007; Pang et al., 2004; Pristera et al., 2012; Russelakis-Carneiro et al., 2004; Tauzin et al., 2008;

382 Waugh, 2013; Waugh et al., 2011a; Waugh et al., 2011b) to generate a composite yet simple

383 signal to assess if there was any redistribution of these structurally related non-sterol molecules

384 in the density gradient following cyclodextrin addition (figure 4). We observed that the

385 ganglioside content of the buoyant fractions was decreased by about $50 \%$ following

386 cyclodextrin treatment and this is consistent with mathematical analysis that vesicle size

387 reduction is due to the non-selective desorption of membrane lipids. 


\section{Discussion}

390

391

392

393

394

395

396

397

398

400

401

402

403

404

405

406

407

408

409

410

411

Our combined biophysical, biochemical, and mathematical analyses demonstrate that cyclodextrin-induced cholesterol extraction can lead to an increase in equilibrium density by inducing membrane shrinkage. The cyclodextrin-induced shift of biomolecules to a denser membrane fraction can be accounted for by a large change in vesicle volume, without necessarily having to evoke the disruption of liquid-ordered membrane microdomains. These new findings have implications for the use of cyclodextrin-induced sterol depletion as a means of assessing whether a protein associates with cholesterol-rich lipid rafts. At high cholesterol levels, such as those reported here in the control buoyant membranes, one might expect significant levels of lipid rafts or even for the entire membrane to exist solely in the liquidordered phase (Almeida et al., 2005; Armstrong et al., 2013; Munro, 2003; Swamy et al., 2006) and hence, removal of cholesterol with cyclodextrin would be predicted to disrupt these rafts (Cabrera-Poch et al., 2004; Kabouridis et al., 2000; Larbi et al., 2004). However, in the context of the type of experiments described here, a cyclodextrin-dependent change in membrane density may only imply that a biomolecule is associated with a cholesterol-rich membrane and does not necessarily report the stable association of that component with lipid raft microdomains.

Our results suggest that at least under the experimental conditions employed here, cyclodextrin-induced reduction of membrane size can also be effected by the extraction of molecules other than sterols. The apparent lack of selectivity for cyclodextrin-induced biomolecule desorption demonstrated here leads us to speculate that these agents could potentially be repurposed to treat a range of conditions similar to Niemann-Pick type C, that feature enlarged endosomal membrane phenotypes due to defective lipid trafficking and/or metabolism but importantly, do not necessarily involve cholesterol accumulation. An example 
412 of a disease to consider in this regard could be oculocerebrorenal syndrome of Lowe (OCRL), a

413 neurodevelopmental condition characterized by phosphatidylinositol 4,5-bisphosphate

414 accumulation on endosomal membranes due to inactivating mutations in the OCRL

415 phosphoinositide 5-phosphatase (reviewed in (Billcliff and Lowe, 2014; Clayton et al., 2013b)).

416 Furthermore, whilst cyclodextrin has a high affinity for sterol lipids it is also known to bind

417 phosphoinositides such as phosphatidylinositol 4-phosphate (Davis et al., 2004), and this

418 further supports the idea that these macromolecules could have applications in the treatment

419 of a number of inherited phospholipid storage disorders. This suggests a new type of drug

420 action involving agents designed to alter membrane surface area through the reduction of

421 membrane mass. The objective of such treatments would be to increase the membrane

422 concentrations of more cyclodextrin-resistant biomolecules, in order to restore or amplify

423 membrane-based signaling or trafficking functions. This has already been shown for the

424 epidermal growth factor receptor, which is subject to augmented levels of constitutive

425 activation following cyclodextrin treatment (Pike and Casey, 2002; Westover et al., 2003).

426 However, these possible uses for cyclodextrin remain speculative and further work is required

427 to investigate if the biophysical changes documented here under specific in vitro conditions also

428 occur on intracellular membranes in live cells.

429 In conclusion, this work throws new light on the mechanism of action of methyl- $\beta$-cyclodextrin

430 on biological membranes. This may lead to a reassessment of its use in cell-based laboratory

431 experiments while at the same time widening its potential applications in the therapeutic arena.

432 In particular, this study indicates that the cholesterol-independent effects of cyclodextrin on

433 membrane area may have more general applications in the treatment of intracellular lipid

434 storage diseases. 


\section{References}

438 Almeida, P.F., Pokorny, A., and Hinderliter, A. (2005). Thermodynamics of membrane domains. Biochim 439 Biophys Acta 1720, 1-13.

440 Armstrong, C.L., Marquardt, D., Dies, H., Kucerka, N., Yamani, Z., Harroun, T.A., Katsaras, J., Shi, A.C., and

441 Rheinstadter, M.C. (2013). The Observation of Highly Ordered Domains in Membranes with Cholesterol. 442 PLoS One 8, e66162.

443 Barylko, B., Mao, Y.S., Wlodarski, P., Jung, G., Binns, D.D., Sun, H.Q., Yin, H.L., and Albanesi, J.P. (2009).

444 Palmitoylation controls the catalytic activity and subcellular distribution of phosphatidylinositol 4-kinase 445 II $\{$ alpha\}. J Biol Chem 284, 9994-10003.

446 Bate, C., Tayebi, M., and Williams, A. (2008). Sequestration of free cholesterol in cell membranes by 447 prions correlates with cytoplasmic phospholipase A2 activation. BMC Biol 6, 8.

448 Billcliff, P.G., and Lowe, M. (2014). Inositol lipid phosphatases in membrane trafficking and human 449 disease. Biochem J 461, 159-175.

450 Blank, N., Schiller, M., Krienke, S., Wabnitz, G., Ho, A.D., and Lorenz, H.M. (2007). Cholera toxin binds to 451 lipid rafts but has a limited specificity for ganglioside GM1. Immunol Cell Biol 85, 378-382.

452 Cabrera-Poch, N., Sanchez-Ruiloba, L., Rodriguez-Martinez, M., and Iglesias, T. (2004). Lipid raft 453 disruption triggers protein kinase $C$ and Src-dependent protein kinase D activation and Kidins220 454 phosphorylation in neuronal cells. J Biol Chem 279, 28592-28602.

455 Camargo, F., Erickson, R.P., Garver, W.S., Hossain, G.S., Carbone, P.N., Heidenreich, R.A., and Blanchard, 456 J. (2001). Cyclodextrins in the treatment of a mouse model of Niemann-Pick C disease. Life Sci 70, 131$457 \quad 142$.

458 Chick, H., and Martin, C.J. (1913). The Density and Solution Volume of some Proteins. Biochem J 7, 9245996.

460 Chu, K.M., Minogue, S., Hsuan, J.J., and Waugh, M.G. (2010). Differential effects of the 461 phosphatidylinositol 4-kinases, PI4KIlalpha and PI4KIIlbeta, on Akt activation and apoptosis. Cell Death 462 Dis 1, e106.

463 Clarke, C.J., Ohanian, V., and Ohanian, J. (2007). Norepinephrine and endothelin activate diacylglycerol 464 kinases in caveolae/rafts of rat mesenteric arteries: agonist-specific role of PI3-kinase. American journal 465 of physiology. Heart and circulatory physiology 292, H2248-2256.

466 Clayton, E.L., Minogue, S., and Waugh, M.G. (2013a). Mammalian phosphatidylinositol 4-kinases as 467 modulators of membrane trafficking and lipid signaling networks. Prog Lipid Res 52, 294-304. 
468 Clayton, E.L., Minogue, S., and Waugh, M.G. (2013b). Phosphatidylinositol 4-kinases and PI4P 469 metabolism in the nervous system: roles in psychiatric and neurological diseases. Mol Neurobiol 47, $470 \quad 361-372$.

471 Correa, J.R., Atella, G.C., Vargas, C., and Soares, M.J. (2007). Transferrin uptake may occur through 472 detergent-resistant membrane domains at the cytopharynx of Trypanosoma cruzi epimastigote forms. 473 Mem Inst Oswaldo Cruz 102, 871-876.

474 Davidson, C.D., Ali, N.F., Micsenyi, M.C., Stephney, G., Renault, S., Dobrenis, K., Ory, D.S., Vanier, M.T., 475 and Walkley, S.U. (2009). Chronic cyclodextrin treatment of murine Niemann-Pick C disease ameliorates 476 neuronal cholesterol and glycosphingolipid storage and disease progression. PLoS One 4, e6951.

477 Davis, A.J., Perera, I.Y., and Boss, W.F. (2004). Cyclodextrins enhance recombinant phosphatidylinositol 478 phosphate kinase activity. J Lipid Res 45, 1783-1789.

479 Dawson, R.M.C., Elliot, D.C., Elliot, W.H., and Jones, K.M. (1986). Data for Biochemical Research, Third 480 edn (Oxford Science Publications).

481 Domon, M.M., Besson, F., Bandorowicz-Pikula, J., and Pikula, S. (2011). Annexin A6 is recruited into lipid 482 rafts of Niemann-Pick type $C$ disease fibroblasts in a Ca2+-dependent manner. Biochem Biophys Res 483 Commun 405, 192-196.

484 Ersek, A., Xu, K., Antonopoulos, A., Butters, T.D., Santo, A.E., Vattakuzhi, Y., Williams, L.M., Goudevenou, 485 K., Danks, L., Freidin, A., et al. (2015). Glycosphingolipid synthesis inhibition limits osteoclast activation 486 and myeloma bone disease. J Clin Invest 125, 2279-2292.

487 Fischer, H., Polikarpov, I., and Craievich, A.F. (2004). Average protein density is a molecular-weight488 dependent function. Protein Sci 13, 2825-2828.

489 Ford, C.P., Stemkowski, P.L., Light, P.E., and Smith, P.A. (2003). Experiments to test the role of 490 phosphatidylinositol 4,5-bisphosphate in neurotransmitter-induced M-channel closure in bullfrog 491 sympathetic neurons. J Neurosci 23, 4931-4941.

492 Fujiki, Y., Hubbard, A.L., Fowler, S., and Lazarow, P.B. (1982). Isolation of intracellular membranes by 493 means of sodium carbonate treatment: application to endoplasmic reticulum. J Cell Biol 93, 97-102.

494 Haynes, W.M. (2005). CRC Handbook of Chemistry and Physics, 94 edn (Taylor \& Francis Group).

495 Heine, T., Dos Santos, H.F., Patchkovskii, S., and Duarte, H.A. (2007). Structure and dynamics of beta496 cyclodextrin in aqueous solution at the density-functional tight binding level. J Phys Chem A 111, 56484975654.

498 Hill, W.G., An, B., and Johnson, J.P. (2002). Endogenously expressed epithelial sodium channel is present 499 in lipid rafts in A6 cells. J Biol Chem 277, 33541-33544.

500 Holtta-Vuori, M., Tanhuanpaa, K., Mobius, W., Somerharju, P., and Ikonen, E. (2002). Modulation of 501 cellular cholesterol transport and homeostasis by Rab11. Mol Biol Cell 13, 3107-3122. 
502 Ilangumaran, S., Arni, S., Chicheportiche, Y., Briol, A., and Hoessli, D.C. (1996). Evaluation by dot503 immunoassay of the differential distribution of cell surface and intracellular proteins in 504 glycosylphosphatidylinositol-rich plasma membrane domains. Anal Biochem 235, 49-56.

505 Kabouridis, P.S., Janzen, J., Magee, A.L., and Ley, S.C. (2000). Cholesterol depletion disrupts lipid rafts 506 and modulates the activity of multiple signaling pathways in T lymphocytes. Eur J Immunol 30, 954-963.

507 Kuziemko, G.M., Stroh, M., and Stevens, R.C. (1996). Cholera toxin binding affinity and specificity for 508 gangliosides determined by surface plasmon resonance. Biochemistry 35, 6375-6384.

509 Larbi, A., Douziech, N., Khalil, A., Dupuis, G., Gherairi, S., Guerard, K.P., and Fulop, T., Jr. (2004). Effects 510 of methyl-beta-cyclodextrin on T lymphocytes lipid rafts with aging. Exp Gerontol 39, 551-558.

511 Li, J., Lu, Y., Zhang, J., Kang, H., Qin, Z., and Chen, C. (2010). PI4KIlalpha is a novel regulator of tumor 512 growth by its action on angiogenesis and HIF-1alpha regulation. Oncogene 29, 2550-2559.

513 Li, J., Zhang, L., Gao, Z., Kang, H., Rong, G., Zhang, X., and Chen, C. (2014). Dual inhibition of EGFR at 514 protein and activity level via combinatorial blocking of PI4KIlalpha as anti-tumor strategy. Protein Cell 5, 515 457-468.

516 Lim, C.H., Schoonderwoerd, K., Kleijer, W.J., de Jonge, H.R., and Tilly, B.C. (2006). Regulation of the cell 517 swelling-activated chloride conductance by cholesterol-rich membrane domains. Acta Physiol (Oxf) 187, 518 295-303.

519 Liu, B., Li, H., Repa, J.J., Turley, S.D., and Dietschy, J.M. (2008). Genetic variations and treatments that 520 affect the lifespan of the NPC1 mouse. J Lipid Res 49, 663-669.

521 Liu, B., Ramirez, C.M., Miller, A.M., Repa, J.J., Turley, S.D., and Dietschy, J.M. (2010). Cyclodextrin 522 overcomes the transport defect in nearly every organ of NPC1 mice leading to excretion of sequestered 523 cholesterol as bile acid. J Lipid Res 51, 933-944.

524 Liu, B., Turley, S.D., Burns, D.K., Miller, A.M., Repa, J.J., and Dietschy, J.M. (2009). Reversal of defective 525 lysosomal transport in NPC disease ameliorates liver dysfunction and neurodegeneration in the npc1-/526 mouse. Proc Natl Acad Sci U S A 106, 2377-2382.

527 Liu, Q., Yao, W.D., and Suzuki, T. (2013). Specific interaction of postsynaptic densities with membrane 528 rafts isolated from synaptic plasma membranes. J Neurogenet 27, 43-58.

529 Liu, T.M., Ling, Y., Woyach, J.A., Beckwith, K., Yeh, Y.Y., Hertlein, E., Zhang, X., Lehman, A., Awan, F., 530 Jones, J.A., et al. (2015). OSU-T315: a novel targeted therapeutic that antagonizes AKT membrane 531 localization and activation of chronic lymphocytic leukemia cells. Blood 125, 284-295.

532 Loftsson, T., and Brewster, M.E. (1996). Pharmaceutical applications of cyclodextrins. 1. Drug 533 solubilization and stabilization. J Pharm Sci 85, 1017-1025.

534 Lu, D., Sun, H.Q., Wang, H., Barylko, B., Fukata, Y., Fukata, M., Albanesi, J.P., and Yin, H.L. (2012). 535 Phosphatidylinositol 4-kinase Ilalpha is palmitoylated by Golgi-localized palmitoyltransferases in 536 cholesterol-dependent manner. J Biol Chem 287, 21856-21865. 
537 Matarazzo, S., Quitadamo, M.C., Mango, R., Ciccone, S., Novelli, G., and Biocca, S. (2012). Cholesterol-

538 lowering drugs inhibit lectin-like oxidized low-density lipoprotein-1 receptor function by membrane raft 539 disruption. Mol Pharmacol 82, 246-254.

540 Mazzone, A., Tietz, P., Jefferson, J., Pagano, R., and LaRusso, N.F. (2006). Isolation and characterization 541 of lipid microdomains from apical and basolateral plasma membranes of rat hepatocytes. Hepatology 542 43, 287-296.

543 Mbua, N.E., Flanagan-Steet, H., Johnson, S., Wolfert, M.A., Boons, G.J., and Steet, R. (2013). Abnormal 544 accumulation and recycling of glycoproteins visualized in Niemann-Pick type $C$ cells using the chemical 545 reporter strategy. Proc Natl Acad Sci U S A 110, 10207-10212.

546 Minogue, S., Chu, K.M., Westover, E.J., Covey, D.F., Hsuan, J.J., and Waugh, M.G. (2010). Relationship 547 between phosphatidylinositol 4-phosphate synthesis, membrane organization, and lateral diffusion of 548 PI4KIlalpha at the trans-Golgi network. J Lipid Res 51, 2314-2324.

549 Munro, S. (2003). Lipid rafts: elusive or illusive? Cell 115, 377-388.

550 Navratil, A.M., Bliss, S.P., Berghorn, K.A., Haughian, J.M., Farmerie, T.A., Graham, J.K., Clay, C.M., and 551 Roberson, M.S. (2003). Constitutive localization of the gonadotropin-releasing hormone (GnRH) receptor 552 to low density membrane microdomains is necessary for GnRH signaling to ERK. J Biol Chem 278, 3159355331602.

554 Nebl, T., Pestonjamasp, K.N., Leszyk, J.D., Crowley, J.L., Oh, S.W., and Luna, E.J. (2002). Proteomic 555 analysis of a detergent-resistant membrane skeleton from neutrophil plasma membranes. J Biol Chem $556277,43399-43409$.

557 Nguyen, H.T., Charrier-Hisamuddin, L., Dalmasso, G., Hiol, A., Sitaraman, S., and Merlin, D. (2007). 558 Association of PepT1 with lipid rafts differently modulates its transport activity in polarized and 559 nonpolarized cells. American journal of physiology. Gastrointestinal and liver physiology 293, G11555601165.

561 Nicholson, A.M., and Ferreira, A. (2009). Increased membrane cholesterol might render mature 562 hippocampal neurons more susceptible to beta-amyloid-induced calpain activation and tau toxicity. J 563 Neurosci 29, 4640-4651.

564 Ottico, E., Prinetti, A., Prioni, S., Giannotta, C., Basso, L., Chigorno, V., and Sonnino, S. (2003). Dynamics 565 of membrane lipid domains in neuronal cells differentiated in culture. J Lipid Res 44, 2142-2151.

566 Paladino, S., Lebreton, S., Tivodar, S., Formiggini, F., Ossato, G., Gratton, E., Tramier, M., Coppey567 Moisan, M., and Zurzolo, C. (2014). Golgi sorting regulates organization and activity of GPI proteins at 568 apical membranes. Nat Chem Biol 10, 350-357.

569 Pang, S., Urquhart, P., and Hooper, N.M. (2004). N-glycans, not the GPI anchor, mediate the apical 570 targeting of a naturally glycosylated, GPI-anchored protein in polarised epithelial cells. J Cell Sci 117, $5715079-5086$. 
572 Pike, L.J., and Casey, L. (2002). Cholesterol levels modulate EGF receptor-mediated signaling by altering 573 receptor function and trafficking. Biochemistry 41, 10315-10322.

574 Pike, L.J., and Miller, J.M. (1998). Cholesterol depletion delocalizes phosphatidylinositol bisphosphate and inhibits hormone-stimulated phosphatidylinositol turnover. J Biol Chem 273, 22298-22304.

576 Pinjari, R.V., Joshi, K.A., and Gejji, S.P. (2006). Molecular electrostatic potentials and hydrogen bonding 577 in alpha-, beta-, and gamma-cyclodextrins. J Phys Chem A 110, 13073-13080.

578 Pontikis, C.C., Davidson, C.D., Walkley, S.U., Platt, F.M., and Begley, D.J. (2013). Cyclodextrin alleviates 579 neuronal storage of cholesterol in Niemann-Pick C disease without evidence of detectable blood-brain 580 barrier permeability. J Inherit Metab Dis 36, 491-498.

581 Pristera, A., Baker, M.D., and Okuse, K. (2012). Association between tetrodotoxin resistant channels and 582 lipid rafts regulates sensory neuron excitability. PLoS One 7, e40079.

583 Ramirez, C.M., Liu, B., Aqul, A., Taylor, A.M., Repa, J.J., Turley, S.D., and Dietschy, J.M. Quantitative role 584 of LAL, NPC2, and NPC1 in lysosomal cholesterol processing defined by genetic and pharmacological 585 manipulations. J Lipid Res 52, 688-698.

586 Ramirez, C.M., Liu, B., Taylor, A.M., Repa, J.J., Burns, D.K., Weinberg, A.G., Turley, S.D., and Dietschy, 587 J.M. (2010). Weekly cyclodextrin administration normalizes cholesterol metabolism in nearly every 588 organ of the Niemann-Pick type C1 mouse and markedly prolongs life. Pediatr Res 68, 309-315.

589 Rodal, S.K., Skretting, G., Garred, O., Vilhardt, F., van Deurs, B., and Sandvig, K. (1999). Extraction of 590 cholesterol with methyl-beta-cyclodextrin perturbs formation of clathrin-coated endocytic vesicles. Mol 591 Biol Cell 10, 961-974.

592 Rosenbaum, A.I., Zhang, G., Warren, J.D., and Maxfield, F.R. (2010). Endocytosis of beta-cyclodextrins is 593 responsible for cholesterol reduction in Niemann-Pick type C mutant cells. Proc Natl Acad Sci U S A 107, 594 5477-5482.

595 Russelakis-Carneiro, M., Hetz, C., Maundrell, K., and Soto, C. (2004). Prion replication alters the 596 distribution of synaptophysin and caveolin 1 in neuronal lipid rafts. Am J Pathol 165, 1839-1848.

597 Shogomori, H., and Futerman, A.H. (2001). Cholesterol depletion by methyl-beta-cyclodextrin blocks 598 cholera toxin transport from endosomes to the Golgi apparatus in hippocampal neurons. J Neurochem $59978,991-999$.

600 Simons, J.P., Al-Shawi, R., Minogue, S., Waugh, M.G., Wiedemann, C., Evangelou, S., Loesch, A., Sihra, 601 T.S., King, R., Warner, T.T., et al. (2009). Loss of phosphatidylinositol 4-kinase 2alpha activity causes late 602 onset degeneration of spinal cord axons. Proc Natl Acad Sci U S A 106, 11535-11539.

603 Spisni, E., Griffoni, C., Santi, S., Riccio, M., Marulli, R., Bartolini, G., Toni, M., Ullrich, V., and Tomasi, V. 604 (2001). Colocalization prostacyclin (PGI2) synthase--caveolin-1 in endothelial cells and new roles for PGI2 605 in angiogenesis. Exp Cell Res 266, 31-43. 
606 Swamy, M.J., Ciani, L., Ge, M., Smith, A.K., Holowka, D., Baird, B., and Freed, J.H. (2006). Coexisting

607 domains in the plasma membranes of live cells characterized by spin-label ESR spectroscopy. Biophys J

$60890,4452-4465$.

609 Swaroop, M., Thorne, N., Rao, M.S., Austin, C.P., McKew, J.C., and Zheng, W. (2012). Evaluation of

610 cholesterol reduction activity of methyl-beta-cyclodextrin using differentiated human neurons and

611 astrocytes. Journal of biomolecular screening 17, 1243-1251.

612 Tauzin, S., Ding, H., Khatib, K., Ahmad, I., Burdevet, D., van Echten-Deckert, G., Lindquist, J.A., Schraven,

613 B., Din, N.U., Borisch, B., et al. (2008). Oncogenic association of the Cbp/PAG adaptor protein with the

614 Lyn tyrosine kinase in human B-NHL rafts. Blood 111, 2310-2320.

615 te Vruchte, D., Speak, A.O., Wallom, K.L., Al Eisa, N., Smith, D.A., Hendriksz, C.J., Simmons, L., Lachmann,

616 R.H., Cousins, A., Hartung, R., et al. (2014). Relative acidic compartment volume as a lysosomal storage

617 disorder-associated biomarker. J Clin Invest 124, 1320-1328.

618 Uesaka, Y., Otsuka, Y., Lin, Z., Yamasaki, S., Yamaoka, J., Kurazono, H., and Takeda, Y. (1994). Simple

619 method of purification of Escherichia coli heat-labile enterotoxin and cholera toxin using immobilized

620 galactose. Microbial pathogenesis 16, 71-76.

621 Vance, J.E., and Karten, B. (2014). Niemann-Pick C disease and mobilization of lysosomal cholesterol by 622 cyclodextrin. J Lipid Res 55, 1609-1621.

623 Vite, C.H., Bagel, J.H., Swain, G.P., Prociuk, M., Sikora, T.U., Stein, V.M., O'Donnell, P., Ruane, T., Ward,

624 S., Crooks, A., et al. (2015). Intracisternal cyclodextrin prevents cerebellar dysfunction and Purkinje cell 625 death in feline Niemann-Pick type C1 disease. Sci Transl Med 7, 276 ra226.

626 Waugh, M.G. (2012). Phosphatidylinositol 4-kinases, phosphatidylinositol 4-phosphate and cancer. 627 Cancer Lett 325, 125-131.

628 Waugh, M.G. (2013). Raft-like membranes from the trans-Golgi network and endosomal compartments. 629 Nat Protoc 8, 2429-2439.

630 Waugh, M.G. (2014). Chromosomal Instability and Phosphoinositide Pathway Gene Signatures in 631 Glioblastoma Multiforme. Mol Neurobiol.

632 Waugh, M.G. (2015). PIPs in neurological diseases. Biochim Biophys Acta 1851, 1066-1082.

633 Waugh, M.G., Chu, K.M., Clayton, E.L., Minogue, S., and Hsuan, J.J. (2011a). Detergent-free isolation and 634 characterization of cholesterol-rich membrane domains from trans-Golgi network vesicles. J Lipid Res $63552,582-589$.

636 Waugh, M.G., Lawson, D., and Hsuan, J.J. (1999). Epidermal growth factor receptor activation is localized 637 within low-buoyant density, non-caveolar membrane domains. Biochem J 337 ( Pt 3), 591-597.

638 Waugh, M.G., Lawson, D., Tan, S.K., and Hsuan, J.J. (1998). Phosphatidylinositol 4-phosphate synthesis in 639 immunoisolated caveolae-like vesicles and low buoyant density non-caveolar membranes. J Biol Chem 640 273, 17115-17121. 
641 Waugh, M.G., Minogue, S., Anderson, J.S., Balinger, A., Blumenkrantz, D., Calnan, D.P., Cramer, R., and 642 Hsuan, J.J. (2003a). Localization of a highly active pool of type II phosphatidylinositol 4-kinase in a $643 \mathrm{p} 97 /$ valosin-containing-protein-rich fraction of the endoplasmic reticulum. Biochem J 373, 57-63.

644 Waugh, M.G., Minogue, S., Blumenkrantz, D., Anderson, J.S., and Hsuan, J.J. (2003b). Identification and 645 characterization of differentially active pools of type llalpha phosphatidylinositol 4-kinase activity in 646 unstimulated A431 cells. Biochem J 376, 497-503.

647 Waugh, M.G., Minogue, S., Chotai, D., Berditchevski, F., and Hsuan, J.J. (2006). Lipid and peptide control 648 of phosphatidylinositol 4-kinase Ilalpha activity on Golgi-endosomal rafts. J Biol Chem 281, 3757-3763.

649 Waugh, M.G., Minogue, S., Clayton, E.L., and Hsuan, J.J. (2011b). CDP-diacylglycerol phospholipid 650 synthesis in detergent-soluble, non-raft, membrane microdomains of the endoplasmic reticulum. J Lipid 651 Res 52, 2148-2158.

652 Welliver, M. (2006). New drug sugammadex: a selective relaxant binding agent. AANA J 74, 357-363.

653 Westover, E.J., Covey, D.F., Brockman, H.L., Brown, R.E., and Pike, L.J. (2003). Cholesterol depletion 654 results in site-specific increases in epidermal growth factor receptor phosphorylation due to membrane 655 level effects. Studies with cholesterol enantiomers. J Biol Chem 278, 51125-51133.

656 Xu, W., Yoon, S.I., Huang, P., Wang, Y., Chen, C., Chong, P.L., and Liu-Chen, L.Y. (2006). Localization of 657 the kappa opioid receptor in lipid rafts. J Pharmacol Exp Ther 317, 1295-1306.

658 Zidovetzki, R., and Levitan, I. (2007). Use of cyclodextrins to manipulate plasma membrane cholesterol 659 content: evidence, misconceptions and control strategies. Biochim Biophys Acta 1768, 1311-1324.

660

661 
662 Legends

663

664 Figure 1.

665 Flow chart outlining the steps involved in the subcellular fractionation procedures, equilibrium 666 density floatation assay and membrane analyses used in the experiments.

667

668 Figure 2.

669 Comparing the effects of cyclodextrin treatment on the biochemical composition of buoyant 670 and dense membrane fractions isolated on equilibrium sucrose density gradients. (A) Change in 671 cholesterol levels as determined by Amplex Red cholesterol assays. Note that there was no 672 significant change in the total amount of cholesterol present in the dense membranes. (B) 673 Levels of the membrane-associated protein PI4KII $\alpha$ were determined by Western blotting and 674 quantitated by image analysis software. Cyclodextrin addition causes a redistribution of PI4KIl $\alpha$ 675 from the buoyant to the dense fractions. Results are presented as mean \pm S.E.M from 676 experiments repeated three times, $* * * p<0.001, * * p<0.01$, NS not statistically significant 677 using the two-tailed student t-test.

678

679 Figure 3.

680 The density of each gradient fraction was determined by refractometry and the conversion of 681 refractive index values to sucrose concentrations was accomplished using Blix tables. Results 682 are presented as mean \pm S.E.M of an experiment repeated three times.

683

684 Figure 4. 
685 Dot blotting of equal volume membrane fractions and detection with HRP-conjugated cholera

686 toxin B subunit was used to determine the levels of ganglioside lipids in control and

687 cyclodextrin-treated membrane fractions. Cyclodextrin addition resulted in a decrease in HRP-

688 conjugated cholera toxin B subunit binding to the buoyant membrane fractions. Results are

689 presented as mean \pm S.E.M from experiments repeated three times.

690

691 Table 1.

692 The size distributions, as measured by dynamic light scattering, of control and cyclodextrin-

693 treated membrane vesicles from different gradient fractions. Results are presented as the mean

$694 \pm$ S.D. of triplicate determinations.

695

696

697 
699 Table 1. Size of membrane vesicles in different gradient fractions following cholesterol

700 depletion with cyclodextrin.

701

\begin{tabular}{ccc}
\hline Treatment & Gradient Fraction & Size (nm) \\
\hline \multirow{2}{*}{ Control } & Buoyant & $779.5 \pm 28.2$ \\
\cline { 2 - 3 } & & Dense \\
\hline \multirow{2}{*}{ Cholesterol Depletion } & Buoyant & $453.0 \pm 177.1$ \\
\cline { 2 - 3 } & & $42.2 \pm 11.5$ \\
& Dense & $270.2 \pm 68.8$ \\
\hline
\end{tabular}

702

703

704

705

706

707

708 


\section{Figure 1 (on next page)}

Flow chart of steps involved in the subcellular fractionation procedures

Figure 1: Flow chart outlining the steps involved in the subcellular fractionation procedures, equilibrium density floatation assay and membrane analyses used in the experiments. 
Harvest cells<smiles>CCCCC</smiles>

Cell disruption by Dounce homogenization

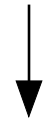

$1000 \mathrm{~g}$ centrifugation for $2 \mathrm{~min}$

Post-1000 g supernatant

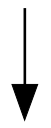

40 to $15 \% \mathrm{w} / \mathrm{v}$ sucrose gradient ultracentrifugation overnight

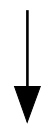

Membrane fraction enriched for trans-Golgi network and endosomes

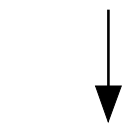

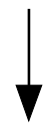

Add sodium carbonate

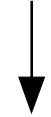

Sonicate<smiles>[CH]1CCC1</smiles>

$40-35-5 \%$ w/v sucrose gradient ultracentrifugation overnight

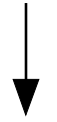

Harvest membrane gradient fractions

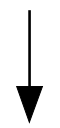

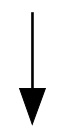

Lipid and Protein Analyses Biophysical Properties

Mathematical Analysis 


\section{2}

Effects of cyclodextrin on vesicle composition

Figure 2: Comparing the effects of cyclodextrin treatment on the biochemical composition of buoyant and dense membrane fractions isolated on equilibrium sucrose density gradients. (A) Change in cholesterol levels as determined by Amplex Red cholesterol assays. Note that there was no significant change in the total amount of cholesterol present in the dense membranes. ( B ) Levels of the membrane - associated protein PI4KIlalpha were determined by Western blotting and quantitated by image analysis software. Cyclodextrin addition causes a redistribution of PI4KIIlalpha from the buoyant to the dense fractions. Results are presented as mean \pm S.E.M from experiments repeated three times, ${ }^{* * *} p<0.001,{ }^{* *} p<$ 0.01 , NS not statistically significant using the two-tailed student t-test. 

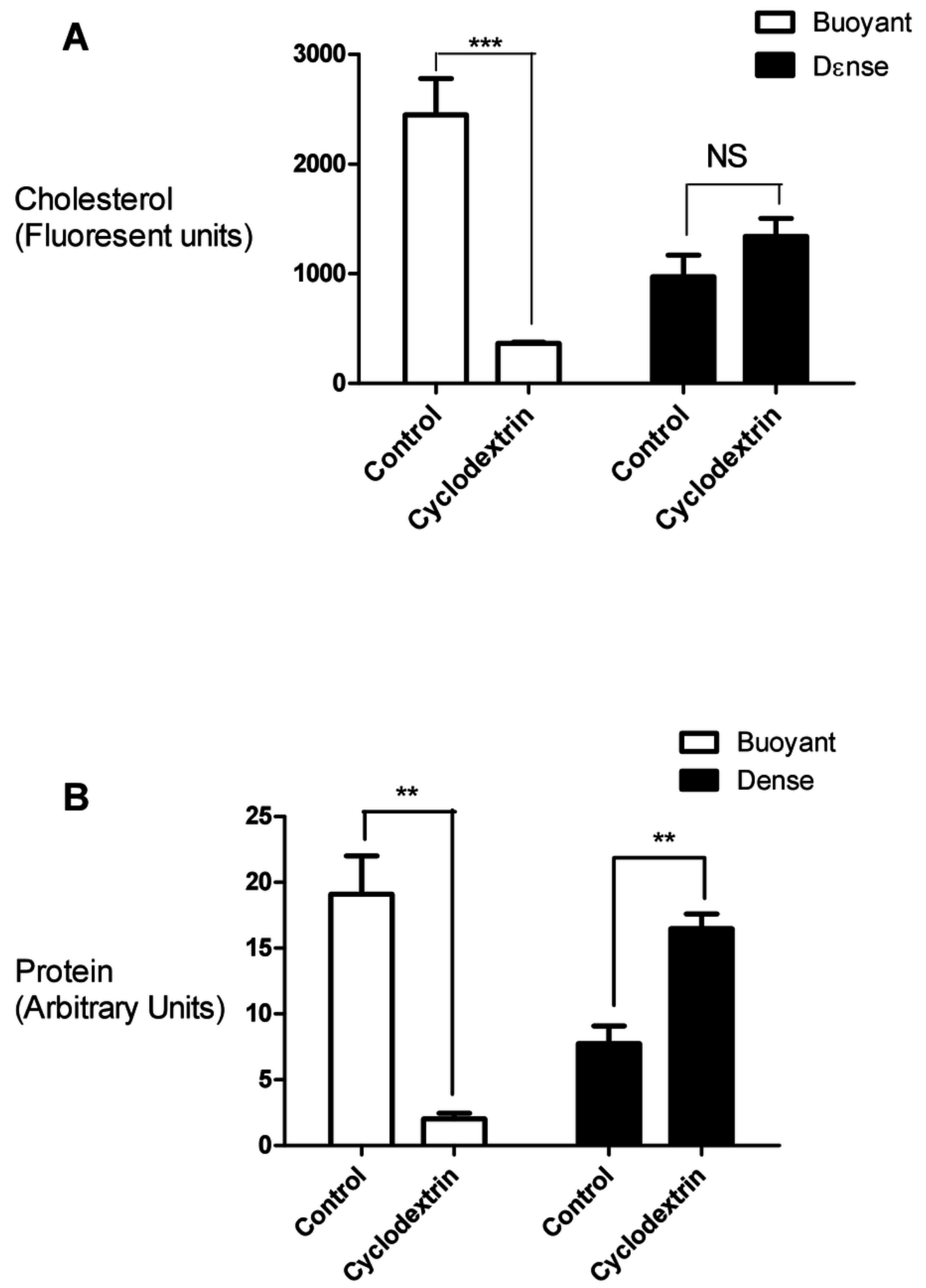
3

Sucrose density gradient profile

Figure 3: The density of each gradient fraction was determined by refractometry and the conversion of refractive index values to sucrose concentrations was accomplished using Blix tables. Results are presented as mean \pm S.E.M of an experiment repeated three times. 


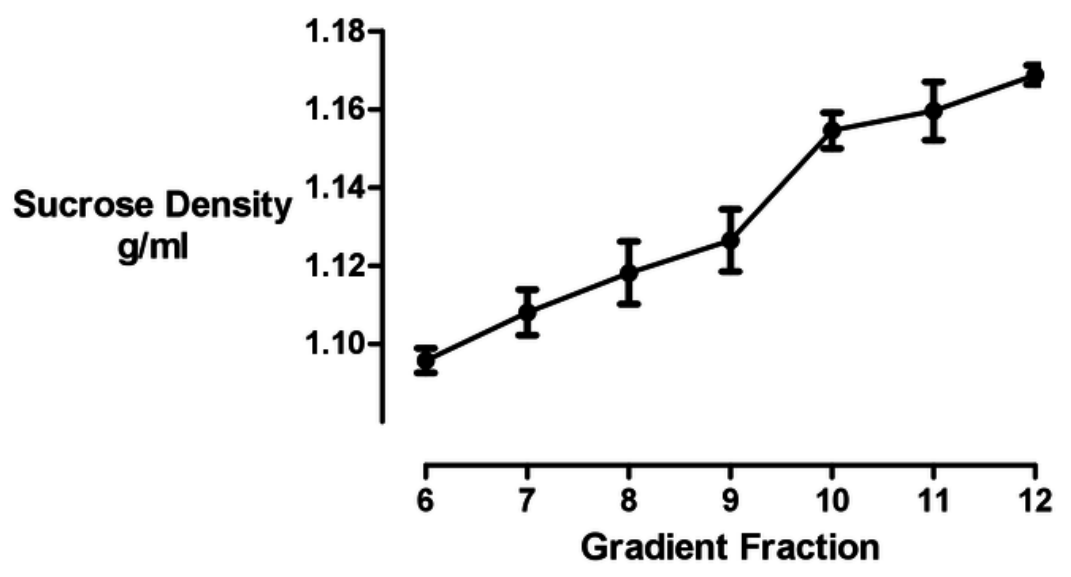




\section{4}

Effect of cyclodextrin on ganglioside distribution profile

Figure 4: Dot blotting of equal volume membrane fractions and detection with HRPconjugated cholera toxin B subunit was used to determine the levels of ganglioside lipids in control and cyclodextrin-treated membrane fractions. Cyclodextrin addition resulted in a decrease in HRP-conjugated cholera toxin B subunit binding to the buoyant membrane fractions. Results are presented as mean \pm S.E.M from experiments repeated three times. 


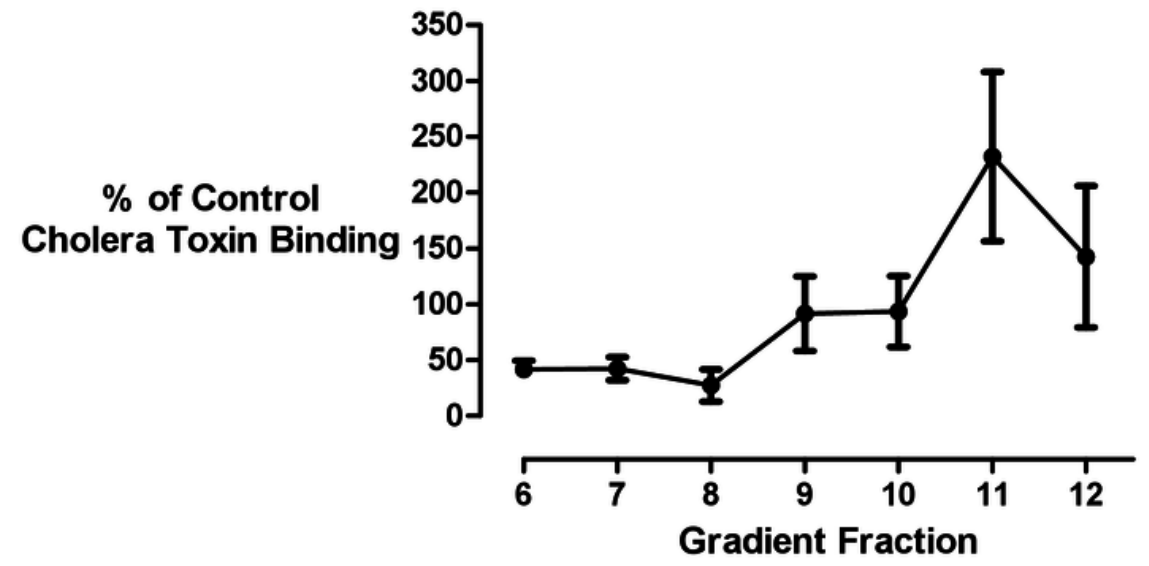




\section{Table $\mathbf{1}$ (on next page)}

Size ofmembrane vesicles in different gradient fraction $\mathrm{s}$ following cholesterol depletion with cyclodextrin.

Table 1: The size distributions, as measured by dynamic light scattering, of control and cyclodextrin-treated membrane vesicles from different gradient fractions. Results are presented as the mean \pm S.D. of triplicate determinations. 


\begin{tabular}{ccc}
\hline Treatment & Gradient Fraction & Size (nm) \\
\hline \multirow{2}{*}{ Control } & Buoyant & $779.5 \pm 28.2$ \\
\cline { 2 - 3 } & & Dense \\
\hline \multirow{2}{*}{ Cholesterol Depletion } & Buoyant & $453.0 \pm 177.1$ \\
\cline { 2 - 3 } & & $42.2 \pm 11.5$ \\
& Dense & $270.2 \pm 68.8$ \\
\hline
\end{tabular}

2 